\title{
ФУНКЦИОНАЛЬНОСТЬ СОВРЕМЕННОЙ САМ-СИСТЕМЫ ДЛЯ 4- И 5-ОСЕВОЙ ОБРАБОТКИ НА ОБРАБАТЫВАЮЩИХ ЦЕНТРАХ С ЧПУ
}

\section{КоНСТаНТИН КАРАБЧЕЕВ}

На примере системы ADEM описан функционал САM-системы, позволяющий реализовывать подготовку управляющих программ для эффективной 4- и 5-осевой обработки сложных деталей на обрабатывающих центрах с ЧПУ.

Современные предприятия промышленности приобретают сложные многокоординатные станки, что накладывает высокие требования к САMсистемам и постпроцессорам, позволяющим получать оптимальные траектории, оптимизировать режимы резания для подобного оборудования.

Ниже приведено несколько примеров необходимой функциональности САМ-системы для подготовки управляющих программ (УП) на обрабатывающие центры с ЧПУ.

\section{ПРИМЕНЕНИЕ 2,5-ОСЕВОГО ФРЕЗЕРОВАНИЯ НА СТАНКАХ С ОСЯМИ ВРАЩЕНИЯ}

Виды обработки:

$\rightarrow$ зонная обработка;

$\rightarrow$ плоская обработка с дополнительной осью вращения.

Один из этапов в многоосевой обработке - это обработка с использованием осей вращения (рис. 1).

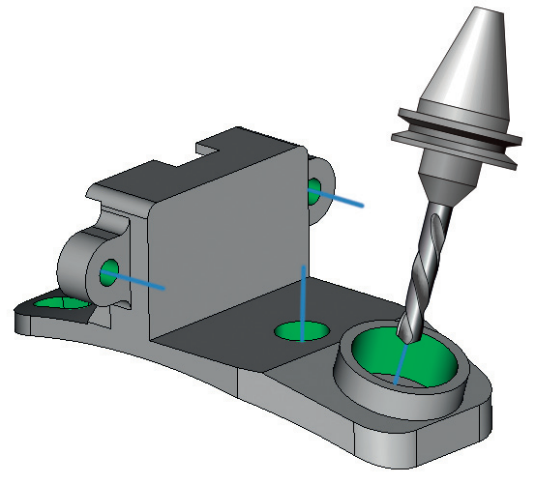

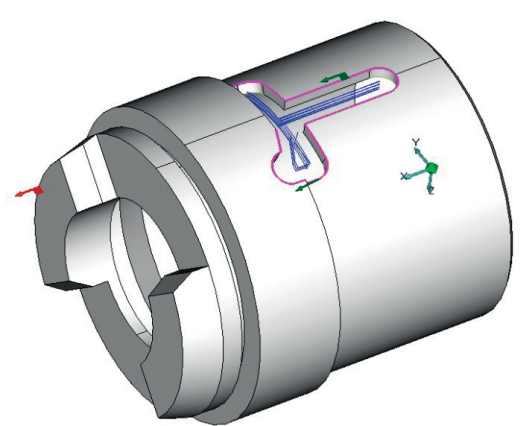

Puc. 2.

Обработка паза по цилиндрической поверхности

К ней относится зонная (позиционная) обработка. Это 2-3-осевая обработка с добавлением позиционирования по осям вращения. Она применяется для производства корпусных деталей, обработка которых может вестись за один установ, без перезакрепления и необходимости использования дополнительной оснастки.

Также использование поворотных осей станка может заменять движение по некоторым линейным осям. В этом случае мы получаем ту же 2,5- или 3-осевую обработку, как бы спроецированную на тело вращения (рис. 2). Этот способ часто применяется для обработки круговых и байонетных пазов, а также при гравировке на цилиндрических поверхностях.

\section{УПРАВЛЕНИЕ ПОЛОЖЕНИЕМ ИНСТРУМЕНТА ОТНОСИТЕЛЬНО ОБРАБАТЫВАЕМОЙ ПОВЕРХНОСТИ}

Параметры управления инструментом:

$\rightarrow$ смещение инструмента; 

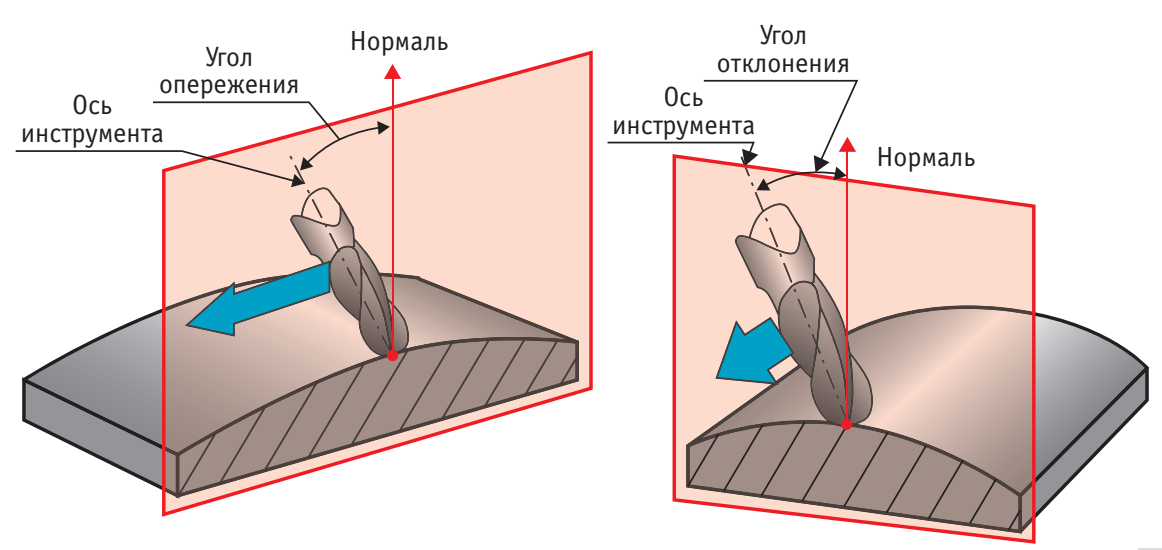

Puc. 4. обработка с учетом кривых и

Puс. 3. Фрезерование с углами опережения и отклонения

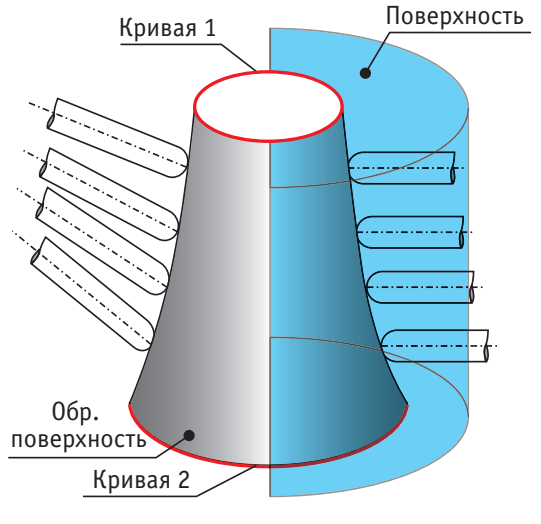

вспомогательных поверхностей $\rightarrow$ углы опережения и отклонения;

$\rightarrow$ управление с помощью кривой и поверхности. При обработке поверхностей часто возникает необходимость корректировки положения инструмента относительно обрабатываемой поверхности. Это может понадобиться при фрезеровании выпуклых поверхностей фрезами с пластинками, когда в центре фрезы образуется участок, без режущих элементов. В таких случаях смещение инструмента в продольном или поперечном направлении позволяет уйти от образования подобных зон с нулевой скоростью резания.

Однако при обработке сильноизогнутых поверхностей не всегда имеется возможность, либо не всегда является эффективным смещение инструмента относительно точки контакта. В этих случаях используется управление осью инструмента по угловым осям (рис. 3). Это использование так называемых углов отклонения и углов опережения, когда инструмент искусственно как бы заваливается вперед по ходу движения.

Для более сложных случаев может быть задействовано управление осью инструмента с использованием дополнительных кривых или поверхностей (рис. 4). В этом случае кончик инструмента идет по обрабатываемой поверхности, а его хвостовая часть движется вдоль указанной кривой. Яркий пример - это обработка моноколес.

\section{ОБРАБОТКА МОНОКОЛЕС}

Основные операции обработки:

$\rightarrow$ плунжерное фрезерование для черновой выборки материала;

$\rightarrow$ чистовая обработка лопастей боковой частью инструмента;

$\rightarrow$ обработка носика лопасти с наклоном инструмента.

При обработке деталей типа «моноколесо» значительное время затрачивается на черновую обработку. Это происходит вследствие того, что моноколеса чаще всего изготавливают из титановых сплавов, а это влияет на подход к обработке. При традиционной технологии черновой обработки таких деталей материал выбирается продольными проходами с небольшим снимаемым припуском и небольшими подачами. Увеличить подачу при такой схеме обработки существенно не получится в связи с высоким коэффициентом износа инструмента. Уменьшить затраты на этом этапе может помочь применение плунжерного фрезерования (рис. 5).

В системе ADEM допустимо использование плунжерного фрезерования не только в 3-осевом режиме, но и совместно с осями вращения.

Этот вид обработки позволяет в два-три раза увеличить подачу за счет использования осевого врезания инструмента и существенно увеличить размер снимаемого припуска. При выполнении проходов система отслеживает положение инструмента относительно обрабатываемых поверхностей, что позволяет уже на первом этапе грубой черновой выборки подготовить криволинейные поверхности к дальнейшей обработке.

При чистовой обработке лопастей моноколеса основная проблема, с которой сталкивается технолог, -

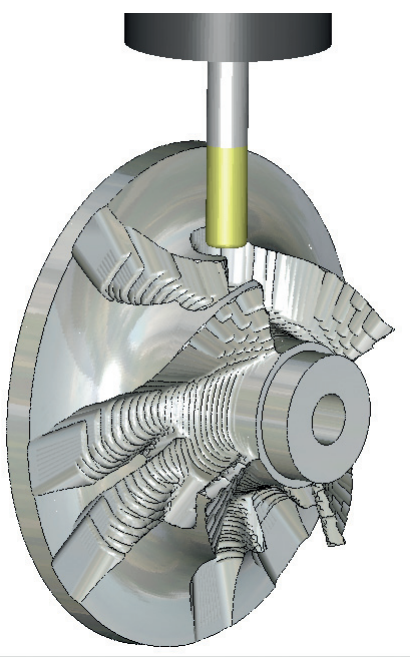

Puc. 5.

Плунжерная обработка межлопаточного пространства 


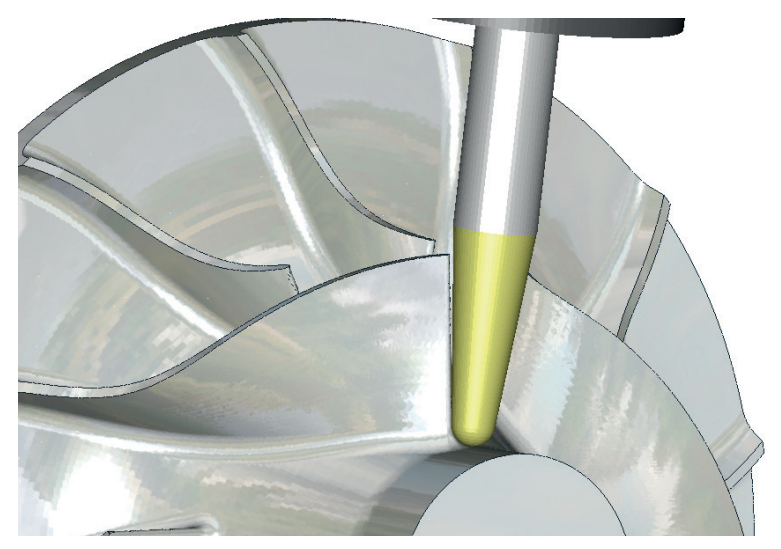

Puc. 6. Чистовая обработка шнека

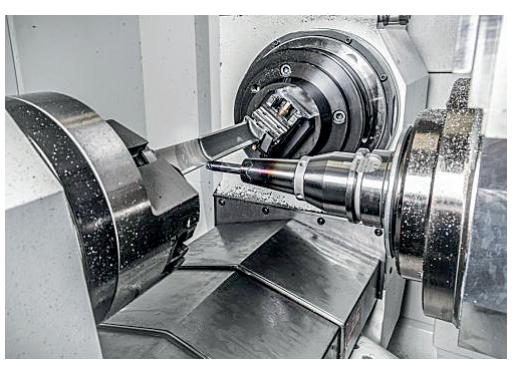

Puc. 7.

Обработка тур-

бинной лопатки

это управление осью инструмента непосредственно на поверхности лопасти и на радиусе скругления (рис. 6).

Как известно, поверхность лопасти представляет собой линейчатую поверхность и теоретически может быть обработана боковой частью обычной концевой фрезы за один проход. В реальности же кривизна втулки и искривление лопасти делает такой подход к обработке невозможным. В производстве, как правило, поверхность лопасти обрабатывают сферическим или сферо-коническим инструментом продольными проходами с постоянным или переменным углом отклонения от поверхности. Но в этом случае при переходе через радиус скругления носика лопасти ось инструмента делает резкий рывок для того, чтобы на следующей поверхности обеспечить заданный угол отклонения. Для устранения такого рода ситуаций в системе ADEM дополнительно к параметрическим средствам управления осью инструмента существует возможность регулировать наклон инструмента с помощью кривых и поверхностей.

\section{ОБРАБОТКА ТУРБИННЫХ ЛОПАТОК}

Основные производственные операции:

$\rightarrow$ спиральная черновая обработка в 4-х координатах с наклоном инструмента;

$\rightarrow$ чистовая обработка поочередно выпуклой и вогнутой частей лопасти.

Традиционный подход к обработке турбинных лопаток заключается в следующем: непрерывная обработка по спирали пера лопатки, затем доработ- ка замковой части (рис. 7). Но этот подход имеет ряд недостатков:

$\rightarrow$ он требует использования сферического инструмента, так как стандартный концевой инструмент будет «нахлопываться» торцем на припуск при переходе с поверхности корыта на поверхность горба, что приведет к поломке;

$\rightarrow$ на радиусах скругления будет грубая огранка, даже при жесткой аппроксимации, причем, чем больше кривизна пера, тем более существенными будут дефекты; кроме того, из-за очень коротких перемещений в кадрах значительно падает реальная подача, что приводит к ускорению износа («засаливанию») инструмента.

В результате ухудшается качество обработки и возрастает доля слесарной доводки.

Для получения качественных поверхностей в системе ADEM присутствует возможность разделять зоны обработки и использовать для каждой зоны оптимальный инструмент.

Обработку поверхностей корыта и радиусов скругления необходимо производить сферическим инструментом с максимально возможным боковым упреждением продольными проходами (рис. 8). Это позволяет: $\rightarrow$ исключить обработку с нулевой скоростью резания; $\rightarrow$ увеличить длину перемещений в кадре;

$\rightarrow$ обработать радиус скругления с точностью станка. Обработку горба пера необходимо производить концевой фрезой с радиусом на торце поперечными проходами с минимальным, но отличным от нуля, продольным угловым упреждением. Это позволяет получить практически зеркальную поверхность.

\section{ОБРАБОТКА ШНЕКОВ}

Проблемы, возникающие при обработке шнеков:

$\rightarrow$ черновая обработка с учетом и без учета оставляемого припуска;

$\rightarrow$ интеллектуальная коррекция положения инструмента.

При обработке деталей типа «шнек» выделяются три типовые задачи, которые приходится решать технологу: $\rightarrow$ черновая обработка межлопастного пространства;

$\rightarrow$ чистовая обработка лопастей;

$\rightarrow$ чистовая обработка втулки шнека.

При черновой обработке межлопастного пространства основная сложность - проход инструмента между соседними лопастями в узких местах. В таких местах часто складывается такая ситуация, когда из-за оставляемого припуска инструмент не может протиснуться между соседними поверхностями. В этом случае система ADEM позволяет проводить инструмент с контролем на зарезания теоретической поверхности, но без учета величины оставляемого припуска. На всех остальных участках траектории припуск будет той величины, которая определена в переходе. 

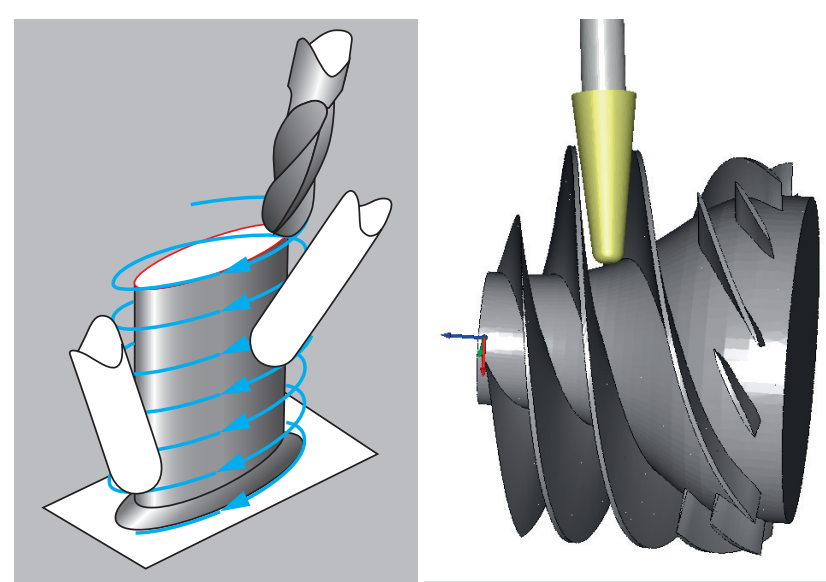

Puc. 8. Спиральная обра- Рис. 9. Моделирование ботка лопатки чистовой обработки шнека

При чистовой обработке поверхности лопасти возникает другая проблема - из-за сильного искривления обработать боковой стенкой инструмента за один проход не удается, а сильно отклонить инструмент от обрабатываемой поверхности, особенно в средней части шнека, где наибольшая высота лопасти, невозможно. В этом случае на помощь приходит интеллектуальная коррекция положения инструмента (рис. 9). Система оценивает каждое положение инструмента и, в случае возникновения коллизии, начинает менять либо ориентацию оси инструмента, либо положение настроечной точки, для того чтобы инструмент смог пройти дальше.

Вариантов обработки втулки шнека существует множество. Главное - проконтролировать наклон инструмента в районе сопряжения боковых поверхностей лопастей и поверхности втулки. Чаще всего для чистовой обработки используют сферический или сферо-конический инструмент. А для управления осью инструмента используют верхние границы соседних лопастей. В этом случае система сама виртуально построит пространственную кривую, равноудаленную от обеих лопастей, и использует ее для управления осью инструмента.

Мы привели лишь несколько примеров 4- и 5-осевой обработки с описанием функциональности необходимой для поддержки эффективной работы современного оборудования. Надеемся в следующих статьях продолжить данную тематику, сосредоточившись не только на схемах обработки и описании оптимизации режимов, но и на автоматическом создании УП по геометрии.

\section{КАРАБЧЕЕВ КоНстантИН -}

ведущий специалист Группы компаний ADEM

\begin{abstract}
Интегрированная CAD/CAM/CAPP система ADEM для автоматизации конструкторскотехнологической подготовки производства. Единый программный комплекс, в состав которого входят модули для: объемного и плоского моделирования; выпуска конструкторской и технологической документации; проектирования техпроцессов; программирования станков с ЧПу (токарных, фрезерных, электроэрозионных, лазерных и др.); управления архивами и проектами; укрупненного трудового нормирования. Содержит средства для анализа технологичности проекта и систему управления справочными данными.
\end{abstract}

\title{
Группа компаний ADEM
}

107497, Москва:

ул. Иркутская, д. 11

тел: +(7) 495 462-0156,

$+(7) 495$ 502-1341

e-mail: moscow@adem.ru
426003, Ижевск:

ул. Красноармейская, д. 69

тел: +(7) 3412 522-341,

+ (7) 3412 522-433

e-mail: izhevsk@adem.ru

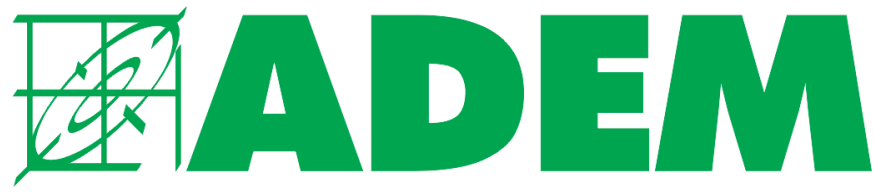

CAD/CAM/CAPP/PDM

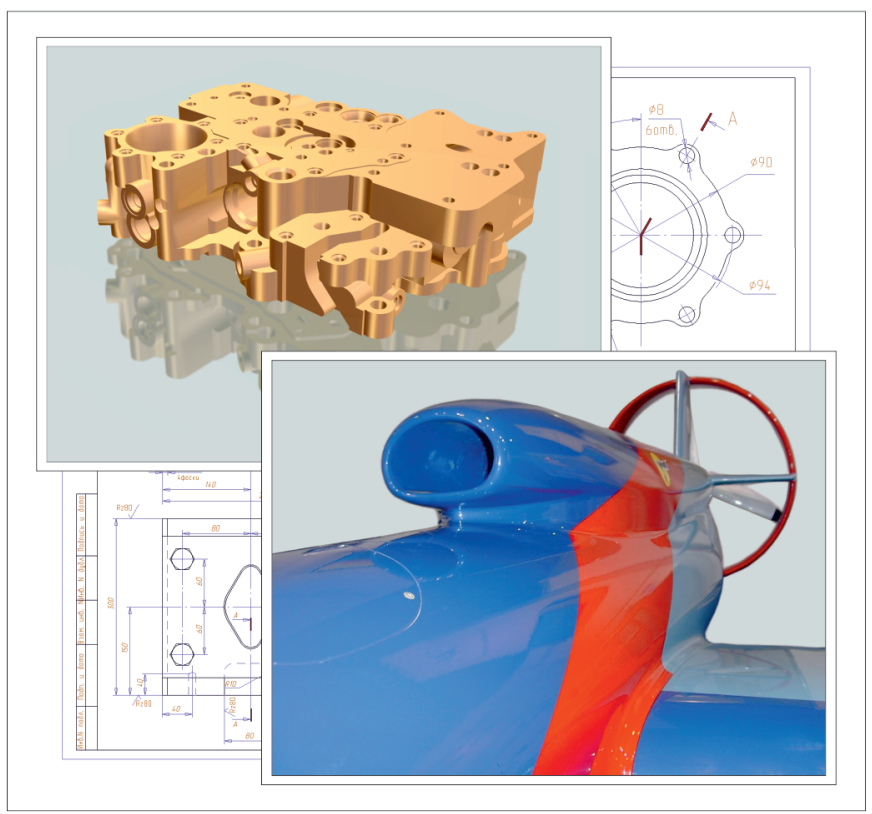

